\title{
Hypoxia-induced secretion of platelet-derived growth factor-BB by hepatocellular carcinoma cells increases activated hepatic stellate cell proliferation, migration and expression of vascular endothelial growth factor-A
}

\author{
YI LU* ${ }^{*}$ NAN LIN*, ZHIJU CHEN and RUIYUN XU \\ Department of Hepatobiliary Surgery, Third Affiliated Hospital of Sun Yat-sen University, \\ Guangzhou, Guangdong 510630, P.R. China
}

Received November 26, 2013; Accepted July 17, 2014

DOI: $10.3892 / \mathrm{mmr} .2014 .2689$

\begin{abstract}
Angiogenesis has an important function in the proliferation and metastasis of hepatocellular carcinoma (HCC) under a hypoxic tumor microenvironment. Activated hepatic stellate cells (HSCs) infiltrate the stroma of liver tumors and potently increase angiogenesis through tumor-stromal interactions, however, the exact mechanism by which this occurs is unknown. The present study aimed to investigate the paracrine effects of $\mathrm{HCC}$-derived platelet-derived growth factor-BB (PDGF-BB) on HSCs under hypoxic conditions. It was demonstrated that PDGF-BB expression was markedly increased in HepG2 cells exposed to hypoxia. Conditioned medium (CM) from HepG2 cells stimulated LX-2 cell proliferation, migration and vascular endothelial growth factor-A (VEGF-A) expression. It was then determined that blocking PDGF-BB expression in HepG2-CM abolished these effects on LX-2 cells. The ectopic expression of PDGF-BB in HepG2 cells strongly affected LX-2 cell proliferation, migration and VEGF-A expression. In conclusion, the present study suggests that hypoxia-induced PDGF-BB secretion by HCC cells stimulates HSCs to accumulate and proliferate in the tumor stroma and the enhanced VEGF-A expression in HSCs may promote $\mathrm{HCC}$ angiogenesis.
\end{abstract}

Correspondence to: Professor Ruiyun $\mathrm{Xu}$, Department of Hepatobiliary Surgery, Third Affiliated Hospital of Sun Yat-sen University, 600 Tianhe Road, Guangzhou, Guangdong 510630, P.R. China

E-mail: 1798010960@qq.com

*Contributed equally

Key words: hepatocellular carcinoma, hepatic stellate cell, platelet-derived growth factor-BB

\section{Introduction}

Primary hepatocellular carcinoma (HCC) is a common malignant tumor and a significant public health concern worldwide (1). Despite the availability of numerous treatment options for patients with HCC, a high rate of recurrence and metastasis results in a low five-year survival rate for this fatal disease (2). There is therefore an urgent requirement to advance our understanding of the mechanisms underlying HCC recurrence and metastasis in order to improve the efficacy of the current treatment strategies. HCC is similar to numerous tumors, has a rich blood supply. HCC relies on the formation of blood vessels for growth and metastasis (3). Although HCC is a highly angiogenic cancer, it is characterized by hypoxia due to its rapid growth rate and numerous hypovascular areas (4). HCC cells are frequently under hypoxic conditions and recent evidence suggests that hypoxia is crucially involved in tumor progression and angiogenesis. The formation of new vessels in the tumor is controlled simultaneously by proand anti-angiogenic factors. The most prominent of several pro-angiogenic factors is vascular endothelial growth factor (VEGF) (5). The upregulation of VEGF expression in tumor tissues has been reported to be associated with poor prognosis in several cancers, including HCC (6).

Previous studies have provided strong evidence that the interaction between tumor cells and their microenvironment, consisting of stromal cells and the extracellular matrix (ECM), contributes to cancer progression $(7,8)$. In the majority of tumors, an abnormal network of stromal cells, growth factors, cytokines and chemokines is critical for the induction of angiogenesis $(9,10)$. The cancer hypoxic microenvironment creates a suitable condition for neo-vascularization by enhancing the expression of pro-angiogenic factors and reducing that of anti-angiogenic factors $(3,11)$. In HCC, VEGF has been reported to be produced by both HCC and stromal cells (10).

The majority of patients with HCC have a history of chronic liver disease, and the presence of liver cirrhosis is closely associated with the development of HCC (12). Activation of hepatic stellate cells (HSCs) is a fundamental step in the development of liver fibrosis and ultimately cirrhosis (13). In addition to these actions, previous studies have indicated that 
activated HSCs infiltrate the liver tumor stroma and become one of the most prominent stromal cell types in the liver tumor environment $(14,15)$. Clinical evidence has revealed that the presence of peritumoral HSCs correlates with the recurrence of HCC (16). Although some data have suggested an important contribution of HSCs to HCC proliferation and metastasis, the exact molecular interactions that occur between these two cell types are unknown. It has been reported that activated HSCs can interact with HCC cells in a paracrine manner $(17,18)$. In the process of liver fibrosis and portal hypertension, activated HSCs have been reported to synthesize pro-angiogenic factors, including VEGF, angiopoietin-1 and several metalloproteinases (19). In experimentally induced liver metastases, activated HSCs have been shown to be pro-angiogenic during the progression of liver cancer (20). These studies suggest that HSCs may contribute to the angiogenic requirement of HCC proliferation and metastasis. The molecular mechanisms of this process remain unclear.

Platelet-derived growth factor (PDGF) is a potent mitogen for mesenchymal cells that is synthesized, stored and released by numerous cell types, including tumor cells (21). PDGF is a dimeric glycoprotein that is composed of two A chains (PDGF-AA), two B chains (PDGF-BB) or a combination of the two (PDGF-AB). Under the hypoxic tumor microenvironment, accumulated evidences indicate that expression of PDGF-BB is elevated in tumor cells and potently stimulates several biological actions in HSCs (22). In colorectal liver metastasis, tumor cell-derived PDGF-BB promotes tumor growth through a growth-promoting effect on HSCs (23). PDGF-BB also affects the angiogenic properties of HSCs during liver fibrosis (24). In liver cancer, however, the function of PDGF-BB in HSCs has not been well characterized.

From these observations, the present study has speculated that HCC-derived factors may influence activated HSCs to regulate angiogenesis under the hypoxic HCC microenvironment. The present study aimed to explore this mechanism through in vitro analysis of the paracrine effects of secreted, HCC-derived PDGF-BB on the proliferation, migration and pro-angiogenic gene expression of HSCs in hypoxia.

\section{Materials and methods}

Cell culture. The HepG2 liver cancer cell line was purchased from the Animal Center Laboratory of Sun Yat-sen University (Guangzhou, China). The LX-2 activated hepatic stellate cell line was obtained from the American Type Culture Collection (ATCC, Manassas, VA, US). All cells were routinely cultured in high-glucose Dulbecco's Modified Eagle's Medium (DMEM) (Gibco-BRL, Grand Island, NY, US) supplemented with 10\% fetal bovine serum (FBS) (HyClone Laboratories, South Logan, UT, USA) at $37^{\circ} \mathrm{C}$ with $5 \% \mathrm{CO}_{2}$ and $95 \%$ air in a humidified incubator (25). The hypoxic stimulation of HepG2 cells was achieved by exposing the cells to $1 \% \mathrm{O}_{2}, 5 \% \mathrm{CO}_{2}$ and $94 \% \mathrm{~N}_{2}$ in an incubator as previously described (26). An indirect co-culture of LX-2 and HepG2 cells was assembled using Transwell membranes (24 mm diameter, $0.4 \mu \mathrm{m}$ pore size; Corning Costar, Acton, MA, USA). A total of $\sim 1 \times 10^{4}$ LX-2 cells were seeded in the lower chamber, and $1 \times 10^{3}$ HepG 2 cells were seeded on the membrane insert. The co-cultures were maintained for $72 \mathrm{~h}$.
Collection of conditioned medium $(C M)$. To prepare the $\mathrm{CM}$, the cells were washed twice with serum-free DMEM one day after being seeded into T75 flasks ( $1 \times 10^{6}$ cells). The cells were incubated with serum-free DMEM under normoxic conditions for $24 \mathrm{~h}$ or under hypoxic conditions for 12 or 24 h. Simultaneously, serum-free DMEM was placed in cell-free culture flasks under the same conditions to serve as a control. For inhibition experiments, CM from HepG2 cells was preincubated with a PDGF-BB neutralizing antibody at a concentration of $5 \mu \mathrm{g} / \mathrm{ml}$ as previously described (27).

Expression constructs and transfection. The expression construct for full-length human PDGF-BB was generated by cloning a polymerase chain reaction-amplifiedPDGF-BB cDNA fragment into the pCDH-CMV-MCS-EF1-copGFP lentiviral vector, which allowed for stable transfection. Virus packaging was performed in HEK293T cells following cotransfection of the recombinant lentiviral expression and packaging plasmids (pGag/Pol, pRev and pVSV-G) using Lipofectamine ${ }^{\mathrm{TM}} 2000$ (Invitrogen Life Technologies, Carlsbad, CA, USA). Target cells were infected with the harvested virus for $12 \mathrm{~h}$, and the original medium was replaced with fresh medium. The cells expressing PDGF-EGFP were sorted by flow cytometry. The lentiviral transduction efficiency was analyzed by western blotting.

MTT assay. The proliferation rate of LX-2 cells was measured using an MTT assay. Briefly, LX-2 cells were seeded in 96-well plates at a density of $2 \times 10^{4}$ cells/well. After $24 \mathrm{~h}, \mathrm{CM}$ was added to the wells, and the plates were incubated at $37^{\circ} \mathrm{C}$ for an additional $24 \mathrm{~h}$. MTT ( $50 \mu \mathrm{l}$ of $5 \mathrm{mg} / \mathrm{ml}$ ) was added to the culture, which was then further incubated for $4 \mathrm{~h}$ at $37^{\circ} \mathrm{C}$. The optical density was measured at $490 \mathrm{~nm}$ using a plate reader. The absorbance values directly correlated with the number of proliferating cells in the culture.

Migration assay. To determine whether the HepG2 cells attracted the LX-2 cells, a migration assay was performed using Transwell chambers containing polycarbonate filters with $8 \mu \mathrm{m}$ pores (Corning Costar). The lower compartment contained CM. The LX-2 cells were harvested and resuspended in DMEM without FBS, at a density of $5 \times 10^{4}$ cells $/ \mathrm{ml}$ and placed in the upper chamber. Following incubation at $37^{\circ} \mathrm{C}$ for $24 \mathrm{~h}$, the filters were collected, and the cells that adhered to the lower surface were fixed, stained for $1 \mathrm{~h}$ with crystal violet (Sigma, St. Louis, MO, USA) in $2 \%$ ethanol and then rinsed in water. The number of cells that migrated across the filters was counted using a Nikon TS100 microscope (Nikon Corporation, Tokyo, Japan) in four high-power fields per insert and average values were calculated. The experiments were performed in triplicate with consistent results.

Western blot analysis. Cells were collected in phosphate-buffered saline (PBS) and lysed on ice for $30 \mathrm{~min}$ in radioimmunoprecipitation assay lysis buffer. The protein content in the cell lysates were quantitated using the Bradford reagent. Equal amounts of protein lysate $(30 \mu \mathrm{g})$ were resolved by $10 \%$ SDS-PAGE and electrophoretically transferred onto polyvinylidene fluoride membranes. The membranes were then blocked in 5\% nonfat dried milk for $1 \mathrm{~h}$. The blots were 
probed with the indicated primary antibodies (PDGF-BB, PDGFR- $\beta$ or VEGF-A) overnight at $4^{\circ} \mathrm{C}$ and then with the appropriate horseradish peroxidase-conjugated secondary antibody $(1: 5,000)$ for $1 \mathrm{~h}$ at room temperature. The blots were developed by enhanced chemiluminescence (Santa Cruz, CA, USA) and exposed to X-ray film. GAPDH was used as a loading control.

ELISA. PDGF-BB is a secreted protein, therefore, the concentration of PDGF-BB in the culture medium was measured to estimate the expression level of PDGF-BB in HepG2 cells. To compare the expression level of PDGF-BB under normoxia and hypoxia, the amount of PDGF-BB in the HepG2 supernatant was analyzed using a commercial ELISA kit (eBioscience, San Diego, CA, USA) according to the manufacturer's instructions.

Statistical analysis. All data are expressed as the mean \pm standard error of the mean, from at least three independent experiments. Statistical analyses were performed using the SPSS statistical software for Microsoft Windows, version 13.0 (SPSS, Inc., Chicago, IL, USA). A two-tailed paired Student's t-test and analysis of variance was used to determine significance between the test and the control conditions. A $\mathrm{P}<0.05$ was considered to indicated a statistically significant difference.

\section{Results}

Effects of hypoxia on PDGF-BB expression in Hep 22 cells. The basal expression of PDGF-BB in HepG2 cells and the change in expression following exposure to hypoxia was analyzed. HepG 2 cells were cultured in an atmosphere containing either $21 \%$ oxygen (normoxia) or $1 \%$ oxygen (hypoxia) for 12 or $24 \mathrm{~h}$. At the end of the incubation, PDGF-BB expression was assessed by western blotting. As shown in Fig. 1A, there was little PDGF-BB expression in HepG2 cells cultured under normoxia. As compared with normoxia, exposure to hypoxia increased the protein expression of PDGF-BB, and this increase was more robust at $24 \mathrm{~h}$ (Fig. 1A). An ELISA was used to detect the secretion of PDGF-BB by HepG2 cells and the PDGF-BB protein concentrations under normoxic and hypoxic conditions were compared. A low concentration of PDGF-BB was detected in the supernatant of HepG2 cells cultured in normoxia, and this concentration significantly increased after exposure to hypoxia (Fig. 1B). These results indicated that PDGF-BB is expressed in HCC cells and is secreted to act in a paracrine manner. Furthermore, the data indicated that hypoxia increased the production and secretion of PDGF-BB by HCC cells.

PDGFR- $\beta$ expression in LX-2 cells increases when co-cultured with HepG2 cells. The activated LX-2 HSC cell line was analyzed for the presence of PDGFR- $\beta$ by western blotting. LX-2 cells were co-cultured with HepG2 cells for $72 \mathrm{~h}$, and the level of PDGF receptor- $\beta$ (PDGFR- $\beta$ ) expression in LX-2 cells grown in monoculture was compared with that of LX-2 cells grown in co-culture. The results confirmed that PDGFR- $\beta$ is expressed in LX- 2 cells grown in monoculture. When co-cultured with HepG2 cells, the protein expression of PDGFR- $\beta$ in LX-2 cells increased (Fig. 2), indicating that HCC cells induced PDGFR- $\beta$ expression in

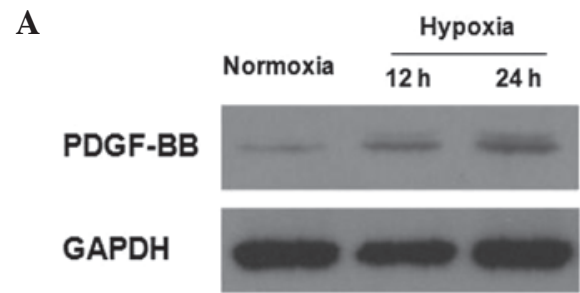

$\mathbf{B}$

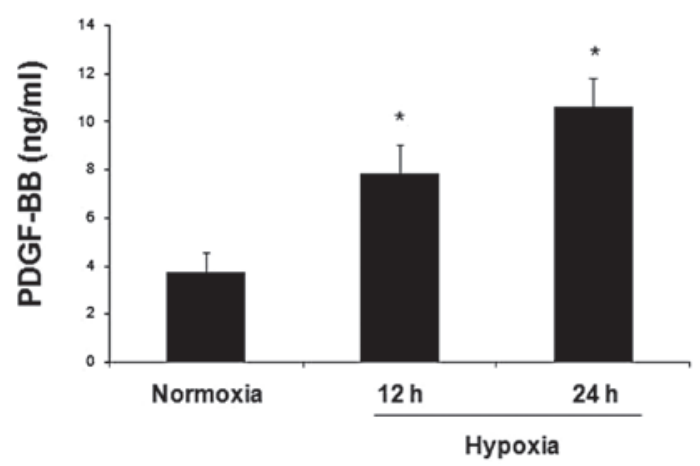

Figure 1. Effect of hypoxia on PDGF-BB expression in HepG2 cells. HepG2 cells were cultured in either normoxia or hypoxia for 12 or $24 \mathrm{~h}$. (A) Following incubation, the expression level of PDGF-BB in HepG2 cells was analyzed by western blotting. There was a clear induction of PDGF-BB expression in HepG2 cells that were cultured in hypoxia. GADPH was used as a loading control. (B) The amount of PDGF-BB that was secreted into the supernatant by the HepG2 cells was measured by ELISA. The highest PDGF-BB concentration was detected in the supernatant of HepG2 cells that were cultured in hypoxia for $24 \mathrm{~h}$. ${ }^{*} \mathrm{P}<0.05$ as compared with normoxia. Error bars represent the means \pm standard error of the mean. PDGF-BB, platelet-derived growth factor-BB.

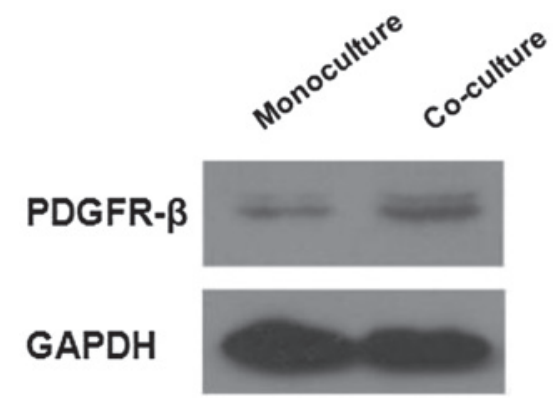

Figure 2. Western blot analysis of PDGFR- $\beta$ protein expression in LX-2 cells in monoculture or co-culture with HepG2 cells. There was a relatively low amount of PDGFR- $\beta$ expression in the LX- 2 cells in monoculture. When cocultured with HepG2 cells, the expression of PDGFR- $\beta$ in the LX-2 cells increased. GAPDH was used as a loading control. PDGFR- $\beta$, platelet-derived growth factor receptor- $\beta$.

activated HSCs. These results suggest that HCC cells affect the biological function of activated HSCs via the paracrine action of PDGF-BB.

Hep $G 2$ cells increased the proliferation and migration of $L X-2$ cells. To examine the effects of HepG2 cells on LX-2 cell proliferation, LX-2 cells were incubated with CM from HepG2 cells cultured under normoxia or hypoxia. An MTT assay was used to measure the proliferation rate of the LX-2 cells. Compared with control medium, HepG2-CM promoted LX-2 cell growth in vitro. HepG2-CM that was collected after culture in hypoxia had a greater effect on LX-2 cell proliferation at various time points (Fig. 3A). 
A

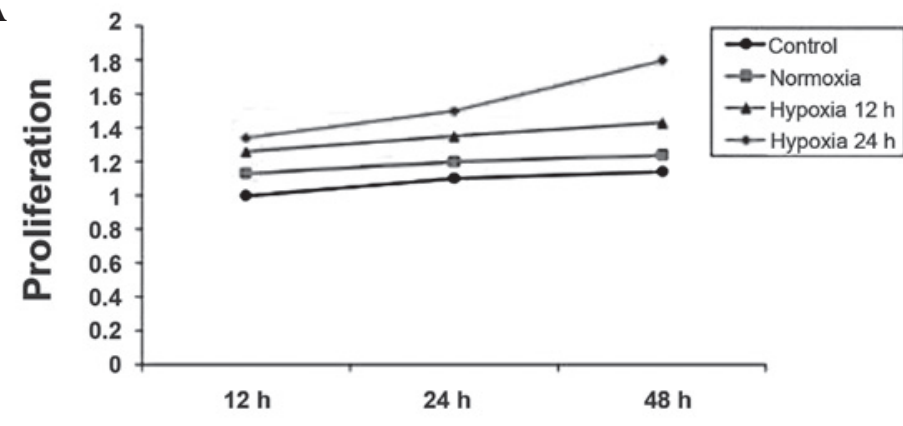

B

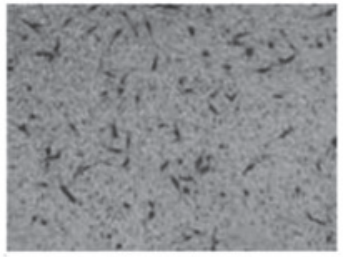

Control

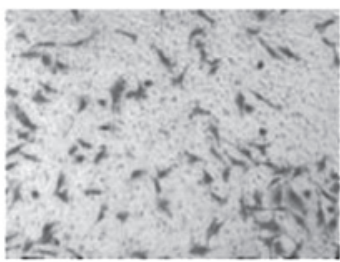

Hypoxia

$12 \mathrm{~h}$

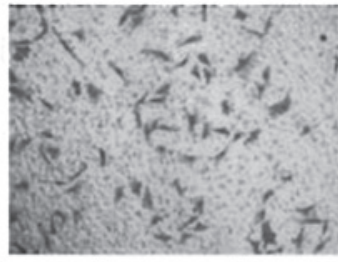

Normoxia

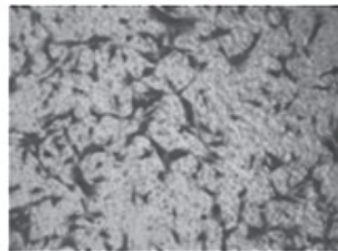

Hypoxia

$24 \mathrm{~h}$

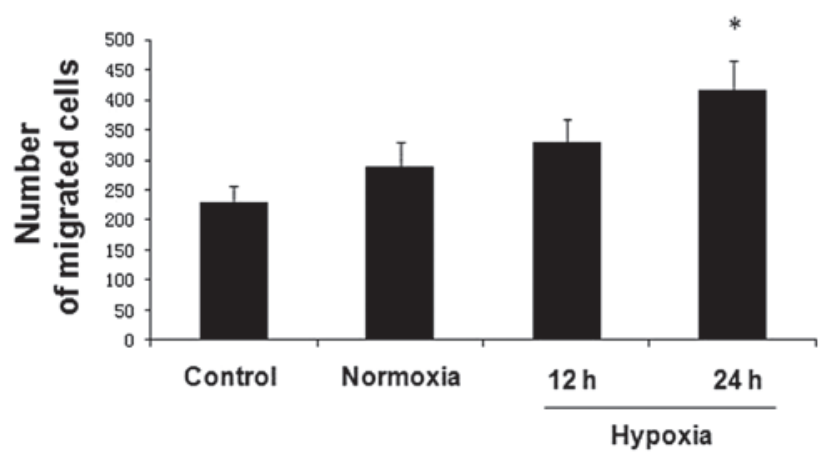

Figure 3. Effect of HepG2 cells on LX-2 cell proliferation and migration. LX-2 cells were cultured in HepG2 conditioned medium, collected from cells grown in normoxia or hypoxia. (A) MTT assays revealed that HepG2 conditioned medium significantly increased LX-2 cell growth in vitro. Conditioned medium collected from cells grown in hypoxia had a greater effect on LX-2 cell proliferation. (B) A cell migration assay was performed to analyze the ability of HepG2 cells to attract LX-2 cells. HepG2 conditioned medium increased the migration of the LX-2 cells, and HepG2 conditioned mediu collected from cells exposed to hypoxia had a greater effect on LX-2 cell migration (original magnification, $\mathrm{x} 40$; stain, crystal violet). Results are expressed as the means \pm standard error of at least three independent experiments. ${ }^{\mathrm{P}}<0.05$ as compared with the control group.

A cell migration assay was used to investigate whether HepG2 cells attracted LX-2 cells. In this experiment, 24-well Transwell plates with $8 \mu \mathrm{m}$ pores were used. A total of $\sim 1 \times 10^{5} \mathrm{LX}-2$ cells were suspended in serum-free medium and placed in the insert. Control or CM that was freshly obtained from HepG2 cells under normoxia or hypoxia was added to the lower chamber. As compared with the control medium, HepG2-CM significantly stimulated the migration of LX-2 cells. HepG2-CM collected from hypoxic culture conditions had a greater effect on LX-2 cell migration than that collected from normoxic culture conditions (Fig. 3B).

HepG2-derived PDGF-BB increases LX-2 cell proliferation and migration. Previous studies have suggested that HepG2 cells increase the proliferation and migration of LX-2 cells and that this effect is greater when $\mathrm{HepG} 2$ cells are exposed to hypoxia. To investigate whether PDGF-BB was causative of these observations, an HepG2 cell line that stably overexpressed PDGF-BB (HepG2-PDGF) was construced. In addition, a PDGF-BB neutralizing antibody was used to inhibit PDGF-BB protein in the HepG2 supernatant, as a negative control. The MTT and cell migration assays were repeated to determine whether HepG2-derived PDGF-BB affects the proliferation and migration of LX-2 cells. As in the previous experiments, the hypoxic $24 \mathrm{~h}$ group was used as the control and the proliferation rate was analyzed at $48 \mathrm{~h}$. The highest PDGF-BB concentration in the HepG2-PDGF supernatant among all of the groups was confirmed by ELISA (data not 
$\mathbf{A}$

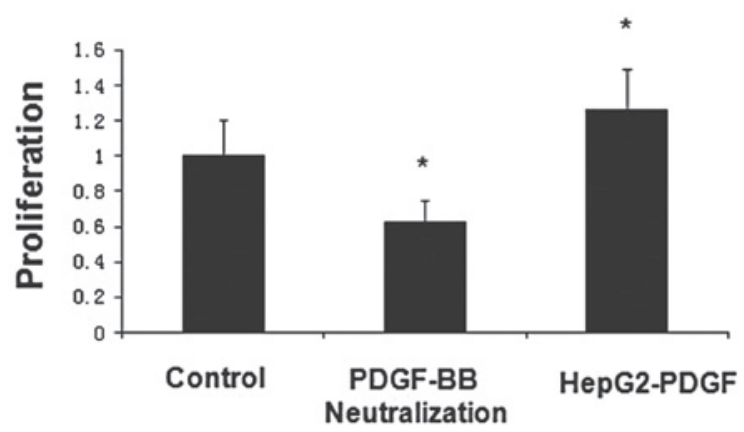

B

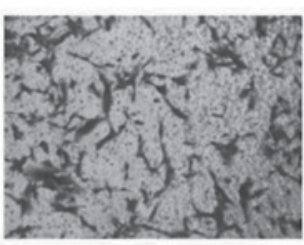

Control

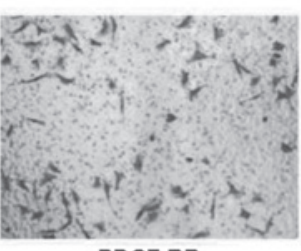

PDGF-BB

Neutralization

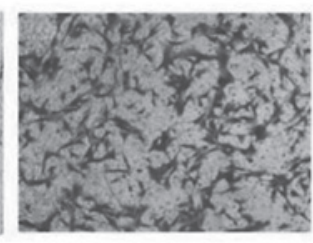

HepG2-PDGF

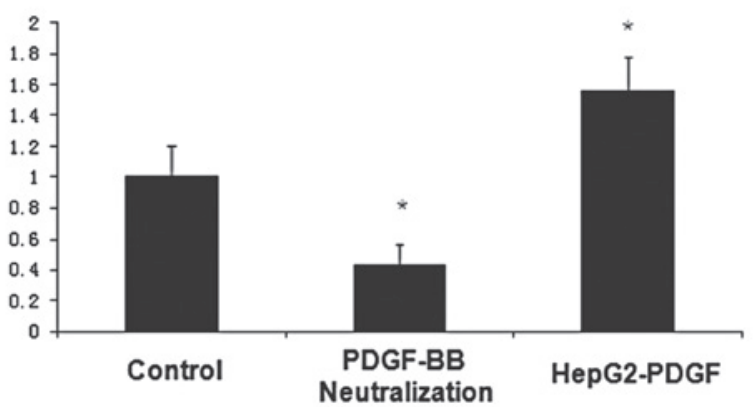

Figure 4. Effect of HepG2-derived PDGF-BB on LX-2 cell proliferation and migration. (A) An MTT assay was performed to ascertain the effect of HepG2-derived PDGF-BB on LX-2 cell proliferation. Conditioned medium from HepG2-PDGF cells significantly increased the proliferation of LX-2 cells. In the presence of the PDGF-BB neutralizing antibody, the effect of HepG2 conditioned medium on LX-2 cell proliferation was reduced. (B) Cell migration assays were performed to analyze the ability of HepG2-derived PDGF-BB to attract LX-2 cells. Conditioned medium, collected from HepG2-PDGF cells, significantly increased LX-2 cell migration in vitro (original magnification, $\mathrm{x} 40$; stain, crystal violet). The number of migrating cells in the control group was set to 1 . Results are expressed as the means \pm standard error of at least three independent experiments. ${ }^{*} \mathrm{P}<0.05$ as compared with the control group. PDGF-BB, platelet-derived growth factor-BB.

shown). The CM from the HepG2-PDGF cells significantly increased LX-2 cell proliferation and migration. The effect of the HepG2-CM on LX-2 cell proliferation and migration was mitigated by the presence of the PDGF-BB neutralizing antibody (Fig. 4A and B). Together, these data suggest that HCC cells stimulate the proliferation of LX-2 cells in a PDGF-BB-dependent paracrine manner.

Effect of HepG2 cells on VEGF-A expression in LX-2 cells. Recent studies have suggested that activated HSCs express pro-angiogenic genes that are involved in intrahepatic angiogenesis in chronic hepatic disease. It was investigated whether HepG2 cells could stimulate LX-2 cells to express VEGF-A, the most prominent protein involved in angiogenesis. LX-2 cells were incubated for $48 \mathrm{~h}$ with CM collected from HepG2 cells cultured under normoxia or hypoxia. The VEGF-A expression in LX-2 cells was determined by western blotting. As compared with the control group, HepG2-CM stimulated the expression of VEGF-A in the LX-2 cells. CM collected from HepG2 cells under hypoxia robustly increased VEGF-A expression in the LX-2 cells (Fig. 5). These results suggest that HCC cells stimulate the pro-angiogenic activity of activated HSCs in a paracrine manner.

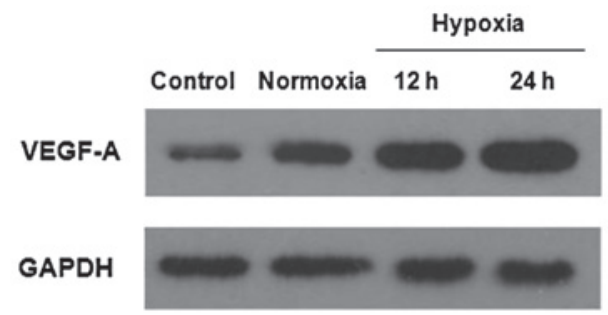

Figure 5. Effect of HepG2 cells on the expression of VEGF-A in LX-2 cells as determined by western blotting. HepG2 cells increased the expression of VEGF-A in LX-2 cells as compared with the control group. Conditioned medium collected from HepG2 cells exposed to hypoxia had a greater effect on VEGF-A expression in LX-2 cells. GAPDH was used as a loading control. VEGF-A, vascular endothelial growth factor A.

HepG2-derived PDGF-BB increases VEGF-A expression in $L X-2$ cells. It was aimed to determine whether PDGF-BB, secreted by HepG2 cells, was responsible for the upregulation of VEGF-A expression in LX-2 cells. LX-2 cells were incubated for $48 \mathrm{~h}$ with $\mathrm{CM}$ collected from HepG2-PDGF cells or with HepG2-CM pre-treated with the PDGF-BB neutralizing antibody. The HepG2-CM from cells exposed to hypoxia for $24 \mathrm{~h}$ was used as a control. Western blot analysis 


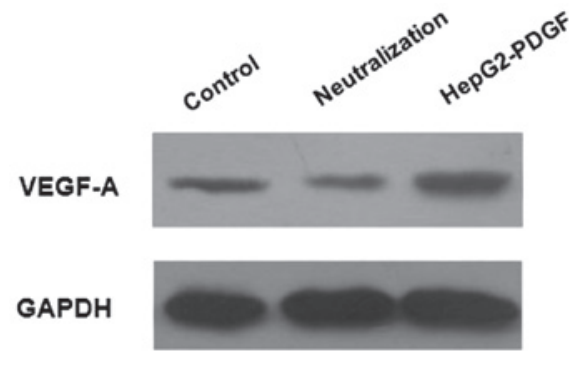

Figure 6. Effect of HepG2-derived PDGF-BB on VEGF-A expression in LX-2 cells as determined by western blotting. Conditioned medium collected from HepG2-PDGF cells significantly increased VEGF-A expression in LX-2 cells. In the presence of the PDGF-BB neutralizing antibody, the ability of HepG2-CM to increase VEGF-A expression in LX-2 cells was inhibited GAPDH was used as a loading control. PDGF, platelet-derived growth factor.

demonstrated that CM from HepG2-PDGF cells significantly increased the expression of VEGF-A in LX-2 cells as compared with the control. In the presence of the PDGF-BB neutralizing antibody, the HepG2-CM-mediated effect on VEGF-A expression in LX-2 cells was diminished (Fig. 6). These results indicated that the PDGF-BB secreted by HCC cells stimulated the pro-angiogenic properties of HSCs.

\section{Discussion}

The present study has demonstrated that hypoxia induces HCC cells to secrete PDGF-BB. PDGF-BB derived from HCC cells can mediate the stimulatory and chemoattractant properties of human HCC cells on HSCs and enhance VEGF-A expression in HSCs. VEGF has been reported to be one of the strongest pro-angiogenic factors for tumor angiogenesis $(5,28)$. These data expand on previous observations that HSCs accumulate in the microenvironment of hepatic tumors, are associated with tumor angiogenesis (15) and promote tumor proliferation and metastasis. The present study confirmed that the PDGF-BB secreted by HCC cells functions in this process, but additional studies are required to clarify the mechanism.

Tumor progression requires the formation of new blood vessels (4). The interactions between tumor and stromal cells are important for the abnormal angiogenesis that occurs in tumors, and these interactions are currently under investigation (10). In the tumor microenvironment, various stromal cells are recruited to and localize within the tumor stroma. The stromal cells and their secreted factors constitute an appropriate microenvironment for tumor angiogenesis (29). Activated HSCs are an important type of stromal cell in HCC. Numerous studies have reported that HSCs accumulate within HCC nodules in patients with liver cirrhosis $(13,30)$. In the present study, CM from HCC cells significantly increased the proliferation and migration of HSCs in vitro. This indicates that HCC cells act in a paracrine manner to attract HSCs and encourage their accumulation within the HCC stroma. Activated HSCs secrete numerous proteins under pathological conditions. These cells have pro-angiogenic properties and interact with endothelial cells that express pro-angiogenic proteins, including VEGF and angiopoietin (19). It was additionally shown that HCC cells increased the expression of VEGF-A in HSCs in vitro, indicating that HSCs in the HCC stroma may participate in tumor angiogenesis.
PDGF-BB is one of five PDGF isoforms, and is a potent mitogen for mesenchymal cells, such as smooth muscle and glial cells (21). Following binding of PDGF-BB to PDGFR, downstream signaling is initiated that leads to cell cycle regulation, migration and differential gene expression (21). PDGFR- $\beta$ is the highest affinity receptor for PDGF-BB (31) and in the process of liver fibrosis, PDGFR- $\beta$ has been reported to be a key marker of HSC activation (30). The present study confirmed that PDGF-BB was expressed in HepG2 cells. The expression of PDGFR- $\beta$ in LX- 2 cells was confirmed, and it was demonstrated that this expression increased when the HSCs were co-cultured together with HCC cells. These results indicate that HCC cells interact with HSCs through the paracrine functions of PDGF-BB. This study demonstrated that PDGF-BB, derived from HCC cells, drives the proliferation, migration and pro-angiogenic protein expression of HSCs. It was speculated that HCC cells secrete PDGF-BB to promote the migration and accumulation of HSCs within the tumor stroma. The induced proliferation and upregulation of VEGF-A expression in HSCs by HCC cell-derived PDGF-BB may create a pro-angiogenic microenvironment that encourages HCC angiogenesis. This process is beneficial for the proliferation and metastasis of HCC. The data from the present study indirectly supports this hypothesis. Since only in vitro data were obtained in this study, additional studies using experimental models of HCC and samples from patients with advanced liver cirrhosis and HCC should be performed to confirm this hypothesis.

Hypoxia often exists during tumor progression due to the rapid tumor growth rate and relative lack of a blood supply (32). To study the paracrine interactions between HSCs and HCC cells, the effects of hypoxia on the paracrine signaling of $\mathrm{HCC}$ cells was examined. The effects of hypoxia on PDGF-BB expression in HCC cells was studied by culturing HCC cells under hypoxic conditions. The concentration of oxygen in the culture atmosphere was $1 \%$ as compared with $20 \%$ in normoxia. This system has been widely used to explore the effect of hypoxia on cells (26). In the present study, evidence was shown supporting that PDGF-BB expression in HCC cells increase with time in hypoxia. Hypoxia is an important regulator of angiogenesis in HCC, but the mechanism has not been fully elucidated. It was found that the expression of VEGF-A in HSCs increased in response to the hypoxia-induced secretion of PDGF-BB by HCC cells. The enhanced VEGF-A expression in HSCs may have an important function in HCC angiogenesis. Future in vivo studies should investigate whether inhibiting PDGF-BB expression in HCC cells, or targeting PDGFR- $\beta$ in HSCs, will affect HCC angiogenesis and reduce the growth and metastasis of this aggressive tumor.

In conclusion, the results of the present study have demonstrated that PDGF-BB secreted by HCC cells promotes the proliferation, migration and VEGF-A expression of HSCs and that hypoxia can affect the expression of PDGF-BB in HCC cells. The data indicate that there is cross-talk between HCC cells and HSCs and the enhanced expression of VEGF-A may affect $\mathrm{HCC}$ angiogenesis. PDGF-BB is an important mediator of this interaction. Although a direct interaction between activated HSCs and HCC cells has not been identified, the shared roles of activated HSCs and HCC cells in tumor progression 
suggest a new therapeutic approach of simultaneously targeting tumor cells and activated HSCs to effectively prevent the development of HCC.

\section{Acknowledgements}

This study was supported by the National Nature Science Foundation of China (grant nos. 81272642 and 81000177) and the Young Teacher Training Program of Sun Yat-sen University (grant no. 11ykpy40).

\section{References}

1. Tang ZY, Ye SL, Liu YK, et al: A decade's studies on metastasis of hepatocellular carcinoma. J Cancer Res Clin Oncol 130: 187-196, 2004

2. Schwartz M, Roayaie S and Konstadoulakis M: Strategies for the management of hepatocellular carcinoma. Nat Clin Pract Oncol 4: 424-432, 2007.

3. Keating GM and Santoro A: Sorafenib: a review of its use in advanced hepatocellular carcinoma. Drugs 69: 223-240, 2009.

4. Coulon S, Heindryckx F, Geerts A, et al: Angiogenesis in chronic liver disease and its complications. Liver Int 31: 146-162, 2011.

5. Kraizer Y, Mawasi N, Seagal J, et al: Vascular endothelial growth factor and angiopoietin in liver regeneration. Biochem Biophys Res Commun 287: 209-215, 2001

6. Schoenleber SJ, Kurtz DM and Talwalkar JA: Prognostic role of vascular endothelial growth factor in hepatocellular carcinoma: systematic review and meta-analysis. Br J Cancer 100: 1385-1392, 2009.

7. Galiè M, Sorrentino C, Montani M, et al: Mammary carcinoma provides highly tumourigenic and invasive reactive stromal cells. Carcinogenesis 26: 1868-1878, 2005.

8. Hernandez-Gea V, Toffanin S, Friedman SL and Llovet JM: Role of the microenvironment in the pathogenesis and treatment of hepatocellular carcinoma. Gastroenterology 144 512-527, 2013.

9. Budhu A, Forgues M, Ye QH, et al: Prediction of venous metastases, recurrence, and prognosis in hepatocellular carcinoma based on a unique immune response signature of the liver microenvironment. Cancer Cell 10: 99-111, 2006.

10. Ribatti D and Vacca A: The role of microenvironment in tumor angiogenesis. Genes Nutr 3: 29-34, 2008.

11. Zhu AX, Duda DG, Sahani DV, et al: HCC and angiogenesis: possible targets and future directions. Nat Rev Clin Oncol 8: 292-301, 2011

12. Guo J and Friedman SL: Hepatic fibrogenesis. Semin Liver Dis 27: 413-426, 2007.

13. Friedman SL: Mechanisms of hepatic fibrogenesis. Gastroenterology 134: 1655-1669, 2008

14. Enzan H, Himeno H, Iwanmura S, et al: Alpha-smooth muscle actin-positive perisinusoidal stromal cells in human hepatocellular carcinoma. Hepatology 19: 895-903, 1994.
15. Thabut D and Shah V: Intrahepatic angiogenesis and sinusoidal remodeling in chronic liver disease: new targets for the treatment of portal hypertension? J Hepatol 53: 976-980, 2010.

16. Liao R, Sun TW, Yi Y, et al: Expression of TREM-1 in hepatic stellate cells and prognostic value in hepatitis B-related hepatocellular carcinoma. Cancer Sci 103: 984-992, 2012.

17. Amann T, Bataille F, Spruss T, et al: Activatd hepatic stellate cells promote tumorigenicity of hepatocellular carcinoma. Cancer Sci 100: 646-653, 2009.

18. Faouzi S, Lepreux S, Bedin C, et al: Activation of cultured rat hepatic stellate cells by tumoral hepatocytes. Lab Invest 79: 485-493, 1999.

19. Olaso E, Salado C, Egilegor E, et al: Proangiogenic role of tumor-activated hepatic stellate cells in experimental melanoma metastasis. Hepatology 37: 674-685, 2003.

20. Heldin CH: Structural and functional studies on platelet-derived growth factor. EMBO J 12: 4251-4259, 1992.

21. Watanabe A, Sohail MA, Gomes DA, et al: Inflammasome-mediated regulation of hepatic stellate cells. Am J Physiol Gatrointest Liver Physiol 296: G1248-G1257, 2009.

22. Bandapalli OR, Macher-Goeppinger S, Schirmacher P, et al: Paracrine signalling in colorectal liver metastases involving tumor cell-derived PDGF-C and hepatic stellate cell-derived PAK-2. Clin Exp Metastasis 29: 409-417, 2012.

23. Aleffi S, Navari N, Delogu W, et al: Mammalian target of rapamycin mediates the angiogenic effects of leptin in human hepatic stellate cells. Am J Physiol Gastrointest Liver Physiol 301: G210-G219, 2011.

24. Jia YL, Shi L, Zhou JN, et al: Epimorphin promotes human hepatocellular carcinoma invasion and metastasis through activation of focal adhesion kinase/extracellular signal-regulated kinase/matrix metalloproteinase-9 axis. Hepatology 54: 1808-1818, 2011.

25. Gleadle JM, Ebert BL, Firth JD, et al: Regulation of angiogenic growth factor expression by hypoxia, transition metals, and chelating agents. Am J Physiol 268: C1362-C1368, 1995.

26. Fingas CD, Bronk SF, Werneburg NW, et al: Myofibroblast-derived PDGF-BB promotes Hedgehog survival signaling in cholangiocarcinoma cells. Hepatology 54: 2076-2088, 2011.

27. Sharma PS, Sharma R and Tyagi T: VEGF/VEGFR pathway inhibitors as anti-angiogenic agents: present and future. Curr Cancer Drug Targets 11: 624-653, 2011.

28. Zhao W, Zhang L, Yin Z, et al: Activated hepatic stellate cells promote hepatocellular carcinoma development in immunocompetent mice. Int J Cancer 129: 2651-2661, 2011

29. Liao R, Wu H, Yi Y, et al: Clinical significance and gene expression study of human hepatic stellate cells in HBV related-hepatocellular carcinoma. J Exp Clin Cancer Res 32: 22-32, 2013.

30. Bowen-Pope DF and Raines EW: History of discovery: platelet-derived growth factor. Arterioscler Thromb Vasc Biol 11: 2397-2401, 2011.

31. Lee UE and Freidman SL: Mechanisms of hepatic fibrogenesis. Best Pract Res Clin Gastroenterol 25: 195-206, 2011.

32. Villanueva A and Llovet JM: Targeted therapies for hepatocellular carcinoma. Gastroenterology 140: 1410-1426, 2011. 